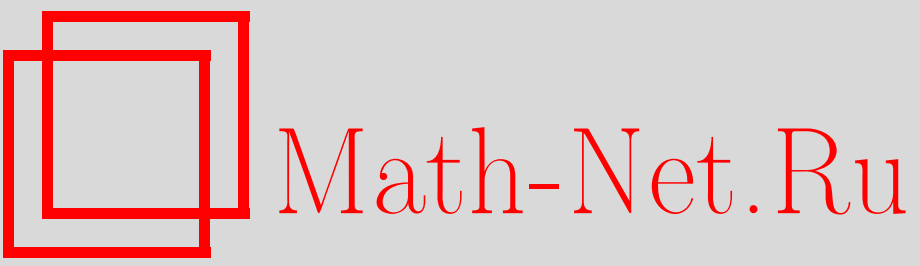

Д. О. Орлов, Производные категории когерентных пучков и мотивы, $У M H, 2005$, том 60, выпуск 6, 231-232

DOI: https://doi.org/10.4213/rm1687

Использование Общероссийского математического портала Math-Net.Ru подразумевает, что вы прочитали и согласны с пользовательским соглашением

http: //www.mathnet.ru/rus/agreement

Параметры загрузки:

IP : 54.197 .130 .99

26 апреля 2023 г., 14:48:21 


\title{
ПРОИЗВОДНЫЕ КАТЕГОРИИ КОГЕРЕНТНЫХ ПУЧКОВ И МОТИВЫ
}

\author{
Д. О. Орлов
}

Ограниченная категория когерентных пучков $\mathbb{D}^{b}(X)$ - это триангулированная категория, которую естественно сопоставить алгебраическому многообразию $X$. Иногда случается, что для двух разных многообразий $X$ и $Y$ имеется эквивалентность $\mathbb{D}^{b}(X) \simeq \mathbb{D}^{b}(Y)$. Возникает естественный вопрос: можно ли в такой ситуации что-то сказать о мотивах данных многообразий? Первый же такой пример [1] - абелево многообразие $A$ и его двойственное $\widehat{A}$ - показывает нам, что мотивы данных многообразий не обязаны быть изоморфны. Однако, по-видимому, что во всех известных примерах их мотивы с рациональными коэффицциентами являются изоморфными.

Напомним определение категории эффективных мотивов Чжоу $\mathrm{CH}^{\mathrm{eff}}(\mathrm{k})$ над полем k. Категория $\mathrm{CH}^{\mathrm{eff}}(\mathrm{k})$ получается как псевдоабелева оболочка (т.е. формальным добавлением коядер всех проекторов) из категории, объекты которой - гладкие проективные схемы над $\mathrm{k}$, а морфизмы из $X$ в $Y$ - это сумма $\bigoplus_{X_{i}} A^{m}\left(X_{i} \times Y\right)$ (по всем компонентам связности $\left.X_{i}\right)$ групп циклов коразмерности $m=\operatorname{dim} Y$ на $X_{i} \times Y$ по модулю рациональной эквивалентности (см. [2], [3]). В [4] В. Воеводский определил триангулированную категорию геометрических мотивов $\mathrm{DM}_{\mathrm{gm}}^{\mathrm{eff}}(\mathrm{k})$. Он стартовал с аддитивной категории $\operatorname{SmCor}(k)$, объекты которой - гладкие схемы конечного типа над k, а морфизмы из $X$ в $Y$ - это свободная абелева группа, порожденная замкнутыми целыми подсхемами $Z \subset X \times Y$, конечными над $X$ и сюръективными над некоторой компонентой связности $X$. Имеется естественное вложение $[-]: \operatorname{Sm}(\mathrm{k}) \rightarrow \operatorname{SmCor}(\mathrm{k})$ категории $\mathrm{Sm}(\mathrm{k})$ гладких схем конечного типа над k. Категория $\operatorname{SmCor}(\mathrm{k})$ аддитивна, и $[X \coprod Y]=[X] \oplus[Y]$. Далее он рассмотрел факторизацию гомотопической категории $\mathscr{H}^{b}(\operatorname{SmCor}(\mathrm{k}))$ ограниченных комплексов по минимальной толстой подкатегории $T$, содержащей все объекты вида $\left[X \times \mathbb{A}^{1}\right] \rightarrow[X]$ и $[U \cap V] \rightarrow[U] \oplus[V] \rightarrow[X]$ для любого открытого покрытия $U \cup V=X$. Триангулированная категория $\mathrm{DM}_{\mathrm{gm}}^{\mathrm{eff}}(\mathrm{k})$ определяется как псевдоабелева оболочка от $\mathscr{H}^{b}(\operatorname{SmCor}(\mathrm{k})) / T$ (см. [4], [3]).

Существует канонический функтор $\mathrm{CH}^{\mathrm{eff}}(\mathrm{k}) \rightarrow \mathrm{DM}_{\mathrm{gm}}^{\mathrm{eff}}(\mathrm{k})$, который является полным вложением, если над полем $k$ существует разрешение особенностей $[4 ; 4.2 .6]$. Таким образом, все, равно в какой из двух категорий (в $\mathrm{CH}^{\mathrm{eff}}(\mathrm{k})$ или в $\left.\mathrm{DM}_{\mathrm{gm}}^{\mathrm{eff}}(\mathrm{k})\right)$ рассматривать мотивы гладких проективных многообразий. Обозначим мотив многообразия $X$ через $\mathrm{M}(X)$, а через $\mathrm{M}(X)_{\mathbb{Q}}$ его мотив в категории мотивов с рациональными коэфффициентами $\mathrm{DM}_{\mathrm{gm}}^{\mathrm{eff}}(\mathrm{k}) \otimes \mathbb{Q}\left(\right.$ и $\left.\mathrm{CH}^{\mathrm{eff}}(\mathrm{k}) \otimes \mathbb{Q}\right)$.

ГиПотеЗА 1. Пусть $X$ и $Y$ - гладкие проективные многообразия и $F: \mathbb{D}^{b}(X) \rightarrow$ $\mathbb{D}^{b}(Y)$ - вполне строгий функтор. Тогда мотив $\mathrm{M}(X)_{\mathbb{Q}}$ является прямым слагаемым в $\mathrm{M}(Y)_{\mathbb{Q}}$. Если $F$ есть әквивалентность, то $\mathrm{M}(X)_{\mathbb{Q}}$ и $\mathrm{M}(Y)_{\mathbb{Q}}$ изоморфны.

В категории $\mathrm{DM}_{\mathrm{gm}}^{\mathrm{eff}}(\mathrm{k})$ имеется тензорное произведение, и $\mathrm{M}(X) \otimes \mathrm{M}(Y)=\mathrm{M}(X \times Y)$. Определим мотив Тейта $\mathbb{Z}(1)$ как образ комплекса $\left[\mathbb{P}^{1}\right] \rightarrow[\operatorname{Spec}(\mathrm{k})]$, сосредоточенного в степенях 2 и 3, и положим $M(p)=M \otimes \mathbb{Z}(1)^{\otimes p}$ для любого мотива $M \in \mathrm{DM}_{\mathrm{gm}}^{\mathrm{eff}}(\mathrm{k})$ и $p \in \mathbb{N}$. Триангулированная категория геометрических мотивов $\mathrm{DM}_{\mathrm{gm}}(\mathrm{k})$ получается из $\mathrm{DM}_{\mathrm{gm}}^{\mathrm{eff}}(\mathrm{k})$ формальным обращением функтора $-\otimes \mathbb{Z}(1)$. Важным и нетривиальным фактом здесь является утверждение, что канонический функтор $\mathrm{DM}_{\mathrm{gm}}^{\mathrm{eff}}(\mathrm{k}) \rightarrow \mathrm{DM}_{\mathrm{gm}}(\mathrm{k})$ является вполне строгим $[4 ; 4.3 .1]$. Более того (см. [4]), для гладких проективных $X$ и $Y$ и любого целого числа $i$ имеется равенство

$$
\operatorname{Hom}_{\mathrm{DM}_{\mathrm{gm}}(\mathrm{k})}(\mathrm{M}(X), \mathrm{M}(Y)(i)[2 i]) \cong A^{m+i}(X \times Y), \text { где } m=\operatorname{dim} Y .
$$

Предположим, что имеется вполне строгий функтор $F: \mathbb{D}^{b}(X) \rightarrow \mathbb{D}^{b}(Y), \operatorname{dim} X=n$, $\operatorname{dim} Y=m$. Этот функтор имеет правый сопряженньй $F^{*}$ по [5], и по теореме 2.2 из [6] (см. также [7; 3.2 .1$]$ ) функтор $F$ представляется объектом на произведении $X \times Y$, т.е. $F \cong \Phi \mathscr{A}$,

Работа выполнена при поддержке РФФИ (грант № 05-01-01034), гранта Президента РФ для поддержки молодых российских ученых (МД-2731.2004.1), Американского фонда гражданских исследований CRDF (RUM1-2661-MO-05) и Фонда содействия отечественной науке. 
где $\Phi_{\mathscr{A}}=\mathbf{R} p_{2 *}\left(p_{1}^{*}(-) \stackrel{\mathrm{L}}{\otimes} \mathscr{A}\right)$ для некоторого $\mathscr{A} \in \mathbb{D}^{b}(X \times Y)$. Каждому функтору вида $\Phi_{\mathscr{A}}: \mathbb{D}^{b}(X) \rightarrow \mathbb{D}^{b}(Y)$ можно сопоставить элемент $a \in A^{*}(X \times Y, \mathbb{Q})$ по правилу

$$
a=p_{1}^{*} \sqrt{\operatorname{td}_{X}} \cdot \operatorname{ch}(\mathscr{A}) \cdot p_{2}^{*} \sqrt{\operatorname{td}_{Y}},
$$

где $\operatorname{td}_{X}$ и $\operatorname{td}_{Y}$ - классы Тодда многообразий $X$ и $Y$. Цикл $a$ имеет смешанный тип, рассмотрим его разложение на компоненты $a=a_{0}+\cdots+a_{n+m}$, где индекс - это коразмерность цикла на $X \times Y$. Каждая компонента $a_{q}$ задает отображение мотивов

$$
\alpha_{q}: \mathrm{M}(X)_{\mathbb{Q}} \rightarrow \mathrm{M}(Y)_{\mathbb{Q}}(q-m)[2(q-m)]
$$

А весь цикл $а$ задает отображение $\alpha: \mathrm{M}(X)_{\mathbb{Q}} \rightarrow \bigoplus_{i=-m}^{n} \mathrm{M}(Y)_{\mathbb{Q}}(i)[2 i]$. Теперь рассмотрим объект $\mathscr{B} \in \mathbb{D}^{b}(X \times Y)$, которьй представляет (левый) сопряженный функтор $F^{*}$, т.е. $F^{*} \cong$ $\Psi_{\mathscr{B}}$, где $\Psi_{\mathscr{B}}=\mathbf{R} p_{1 *}\left(p_{2}^{*}(-) \otimes \mathscr{\mathbf { L }} \mathscr{B}\right)$. Сопоставим объекту $\mathscr{B}$ цикл $b=b_{0}+\cdots+b_{n+m}$ по той же самой формуле (1). Цикл $b$ индуцирует отображение $\beta: \bigoplus_{i=-m}^{n} \mathrm{M}(Y)_{\mathbb{Q}}(i)[2 i] \rightarrow \mathrm{M}(X)_{\mathbb{Q}}$. Так как функтор $\Phi_{\mathscr{A}}$ вполне строгий, то композиция $\Psi_{\mathscr{B}} \circ \Phi_{\mathscr{A}}$ изоморфна тождественному функтору. А применяя теорему Римана-Роха-Гротендика, получаем, что и композиция

$$
\mathrm{M}(X)_{\mathbb{Q}} \stackrel{\alpha}{\rightarrow} \bigoplus_{i=-m}^{n} \mathrm{M}(Y)_{\mathbb{Q}}(i)[2 i] \stackrel{\beta}{\rightarrow} \mathrm{M}(X)_{\mathbb{Q}}
$$

является тождественной, т.е. $\mathrm{M}(X)_{\mathbb{Q}}-$ прямое слагаемое в $\bigoplus_{i=-m}^{n} \mathrm{M}(Y)_{\mathbb{Q}}(i)[2 i]$.

Предположим теперь, что $\operatorname{dim} X=\operatorname{dim} Y=n$ и что носитель объекта $A$ также имеет размерность $n$. Значит, $a_{q}=0$ при $q=0,1, \ldots, n-1$, т.е. $a=a_{n}+\cdots+a_{2 n}$. Нетрудно видеть, что в этом случае и $b=b_{n}+\cdots+b_{2 n}$. Отсюда следует, что композиция $\beta \cdot \alpha: \mathrm{M}(X)_{\mathbb{Q}} \rightarrow \mathrm{M}(X)_{\mathbb{Q}}$, которая является тождественной, совпадает с композицией $\beta_{n} \cdot \alpha_{n}$. И, значит, $\mathrm{M}(X)_{\mathbb{Q}}$ является прямым слагаемым в $\mathrm{M}(Y)_{\mathbb{Q}}$. Более того, в данной ситуации циклы $a_{n}$ и $b_{n}$ целочисленные. Следовательно, и целочисленньй мотив $\mathrm{M}(X)$ является прямым слагаемым в $\mathrm{M}(Y)$.

Теорема 1. Пусть $X$ и $F: \mathbb{D}^{b}(X) \rightarrow \mathbb{D}^{b}(Y)$ вполне строгий функтор такой, что размерность носителя обгекта $\mathscr{A}$, представляющего функтор $F$, равна п. Тогда мотив $\mathrm{M}(X)$ является прямым слагаемым мотива $\mathrm{M}(Y)$. Если к тому же функтор $F$ есть әквивалентность, то мотив в $\mathrm{M}(X)$ и $\mathrm{M}(Y)$ изоморфны.

Примеры таких функторов известны, они приходят из бирационалњной геометрии (см., например, [7]). В этих примерах одна из компонент $\operatorname{supp}(\mathscr{A})$ задает бирациональное отображение $X \rightarrow Y$. Раздутия и антифлипы дают вполне строгие функторы, а флопы - эквивалентности. Отметим, что изоморфизм мотивов влечет изоморфизм всех реализаций.

Для произвольной эквивалентности $\Phi_{\mathscr{A}}: \mathbb{D}^{b}(X) \rightarrow \mathbb{D}^{b}(Y)$ отображение мотивов $\alpha_{n}: \mathrm{M}(X)_{\mathbb{Q}}$ $\rightarrow \mathrm{M}(Y, \mathbb{Q})$, заданное циклом $a_{n} \in A^{n}(X \times Y, \mathbb{Q})$, не обязано быть изоморфизмом. Однако вполне возможно, что верна следующая гипотеза, уточняющая гипотезу 1.

ГиПотеЗА 2. Пусть $\mathscr{A}$ - облект на $X \times Y$, для которого $\Phi_{\mathscr{A}}: \mathbb{D}^{b}(X) \rightarrow \mathbb{D}^{b}(Y)$ есть әквивалентность. Тогда найдутся линейные расслоения $L$ и $M$ на $X$ и $Y$ соответственно такие, что компонента $a_{n}^{\prime}$ облекта $\mathscr{A}^{\prime}:=p_{1}^{*} L \otimes \mathscr{A} \otimes p_{2}^{*} M$ задает изоморфизм между мотивами $\mathrm{M}(X)_{\mathbb{Q}}$ u $\mathrm{M}(Y)_{\mathbb{Q}}$.

Автор благодарен Ю. И. Манину за полезные обсуждения.

\section{СПИСОК ЛИТЕРАТУРЫ}

[1] S. Mukai // Nagoya Math. J. 1981. V. 81. P. 153-175. [2] Ю. И. Манин // Матем. сб. 1968. T. 77. № 4. C. 475-507. [3] S. Bloch // Proc. Sympos. Pure Math. 1997. V. 62. Part 1. P. 329-359. [4] V. Voevodsky // Ann. of Math. Stud. 2000. V. 143. P. 188-238. [5] A. Bondal, M. Van den Bergh // Mosc. Math. J. 2003. V. 3. № 1. P. 1-36. [6] D. Orlov // J. Math. Sci. (New York). 1997. V. 84. № 5. P. 1361-1381. [7] Д. О. Орлов // УМН. 2003. T. 58. № 3. С. 89-172. 